\title{
Shared genetic susceptibility of Type 1 (insulin-dependent) and Type 2 (non-insulin-dependent) diabetes mellitus: contributions of HLA and haptoglobin
}

\author{
S.S. Rich ${ }^{1,2}$, S. S.Panter ${ }^{1}$, F. C. Goetz ${ }^{3}$, B.Hedlund ${ }^{4}$ and J. Barbosa ${ }^{3}$ \\ ${ }^{1}$ Department of Laboratory Medicine and Pathology, ${ }^{2}$ Institute of Human Genetics, and ${ }^{3}$ Division of Endocrinology and Metabolism, \\ Department of Medicine, University of Minnesota, ${ }^{4}$ Biomedical Frontiers, Inc., Minneapolis, Minnesota, USA
}

\begin{abstract}
Summary. Epidemiologic data suggest that having a parent with Type 2 (non-insulin-dependent) diabetes mellitus increases the risk for Type 1 (insulin-dependent) diabetes in siblings of a Type 1 diabetes proband. This increase in risk is consistent with a shared genetic susceptibility between Type 1 diabetes and Type 2 diabetes. We contrast genetic risk factors in three sets of families, consisting of (1) a single Type 1 diabetic child (proband) and non-diabetic parents, (2) multiple Type 1 diabetic siblings and non-diabetic parents, and (3) at least one Type 1 diabetic child and at least one Type 2 diabetic parent. Previous studies have demonstrated that HLA region genes, which elevate the risk in Type 1 diabetes, have no significant effect with respect to the risk for developing Type 2 diabetes. An earlier report cited a contribution by the haptoglobin locus to genetic susceptibility for Type 2 diabetes. We provide evidence that a high risk
\end{abstract}

HLA antigen (HLA-DR3) is decreased to a greater extent in Type 1 patients with a Type 2 parent than in Type 1 patients in which the parents are not diabetic. The role of HLA-DR4 is maintained in these families, with an unexpectedly significant increased rate of transmission of the HLA-DR4 allele from Type 2 parent to Type 1 offspring. The role of haptoglobin in these families does not appear to be important, either with respect to association with diabetes or with respect to linkage with a secondary susceptibility locus. These results indicate that families with a Type 2 parent and Type 1 child, heavily determined by HLA-DR4 linked factors, may represent a homogeneous subset of diabetes susceptibility.

Key words: Genetics, Type 1 (insulin-dependent) diabetes mellitus, Type 2 (non-insulin-dependent) diabetes mellitus, HLA, haptoglobin.
Insight into the pathogenesis of both Type 1 (insulin-dependent) diabetes mellitus and Type 2 (non-insulin-dependent) diabetes is compromised by their unresolved modes of inheritance. The most powerful predictor of risk for developing either Type 1 or Type 2 diabetes is being an identical (monozygotic, or MZ) twin of a diabetic. The $\mathrm{MZ}$ twin concordance for Type 1 diabetes is significantly less than $100 \%$, ranging from $25 \%-50 \%[1,2]$. This decreased $\mathrm{MZ}$ twin concordance rate would imply that nonfamilial environmental factors play an important role in defining an individual's susceptibility to Type 1 diabetes. The "random" environmental factors themselves may be heterogeneous (prenatal, perinatal, cultural), and further obscure the mode of genetic transmission. Heterogeneous environmental factors (environmental "triggers", such as viruses or exposures to pathogens) could effectively mask single gene effects. On the other hand, the MZ twin concordance rate for Type 2 diabetes is close to $100 \%$ [3], indicating that the susceptibility is highly familial, with Type 2 diabetes determined by genetic and/or common environmental factors.
Although individual estimates of risk to relatives for Type 1 and Type 2 diabetes are variable, depending upon ethnic origin and clinical manifestation of disease, there is a clear relationship between the magnitude of diabetic risk with the degree of genetic relationship of the proband $[4,5]$. As the degree of relationship decreases from MZ twin $(100 \%$ of genes shared identical by descent), to firstdegree relatives (full siblings, parents and offspring; $50 \%$ shared), to second degree ( $25 \%$ shared) and third degree (12.5\% shared) relatives, the risks for Type 1 and Type 2 diabetes decrease in a non-linear fashion. Under the assumption of no dominance, supported by equivalent risks to siblings and parents, single locus models predict a linear relationship between diabetic risk and the percent of genes shared identical by descent. The single locus models fail to account for the non-linear nature of familial risk for Type 1 and Type 2 diabetes [6-9]. The degree of reduced penetrance required for a single locus to account for risks in distant relatives is difficult to reconcile with the observed $\mathrm{MZ}$ twin concordance rates for both Type 1 and Type 2 diabetes. The implication from these data is that 
additional loci contributing to the genetic susceptibility for both Type 1 and Type 2 diabetes are required.

In this report, we focus on the susceptibility to Type 1 diabetes in families distinguished by disease status of the parents of a Type 1 diabetic offspring. In one set of families, parents of the Type 1 proband are disease-free. In another set of families, at least one parent of the Type 1. proband has Type 2 diabetes. Family-type specific differences in relative risk or linkage relationships would represent evidence that locus-specific differences exist which relate to the Type 2 diabetes susceptibility in parents. We present evidence that HLA-DR 4 or a factor in linkage disequilibrium or restricted by HLA-DR4 may contribute to the increased susceptibility of Type 1 diabetes in families with Type 2 diabetic parents.

\section{Subjects and methods}

\section{Subjects}

The families studied in this report consist of three types, distinguished by disease status in the parents (Type 2 diabetic or non-diabetic) and by the number of offspring with Type 1 diabetes (one, or more than one). Families designated as "pure Type 1 diabetes" have at least one offspring with Type 1 diabetes and both parents phenotypically normal. Within this group, those families with one Type 1 diabetic offspring (the proband) are termed "simplex", while those with the proband and at least one Type 1 diabetic sibling are termed "multisib". The "Type 1/Type 2" families have at least one offspring with Type 1 diabetes and at least one parent with Type 2 diabetes.

All definitions of the diabetic state are made according to the criteria of the National Diabetes Data Group [10]. From our database of Type 1 families with HLA and haptoglobin typing data, we ascertained 98 simplex families (normal parents and one Type 1 diabetic offspring), 36 multisib families (normal parents and two or more Type 1 offspring), and 28 Type 1 /Type 2 families (at least one Type 2 parent and one or more Type 1 offspring).

This study was approved by the University of Minnesota Committee on the Use of Human Subjects in Research, and each individual signed a consent form upon admission to the University of Minnesota General Clinical Research Center.

\section{Experimentalmeasurements}

Typing for HLA and haptoglobin were performed in an unbiased, blinded protocol without regard to presence of Type 2 diabetes in parents or by number of Type 1 diabetes in offspring. In the 28 Type 1/Type 2 families, HLA-A and HLA-B typing data were available for 24 and HLA-DR typing data were available for 18 families. HLA data were available for all 98 Type 1 simplex families and 36 Type 1 multisib families. Control data for HLA antigens were provided from the population of donors (617 for HLA-A, HLA-B and 511 for HLA-DR) at the University of Minnesota Hospitals for the Eighth International Workshop as previously reported [11]. Control data for haptoglobin typing were provided from 168 unrelated nondiabetic individuals [12].

HLA-A, HLA-B, and HLA-DR antigens were determined on all available family members. All HLA typing was performed in the HLA laboratories of the University of Minnesota Hospitals and Clinics by published methods [13]. Haptoglobin typing was performed using plasma in a modification of the method of Roy [14]. This modification preferentially keeps haptoglobin soluble while precipitating other plasma proteins. Purified 1-1 haptoglobin (Biomedical Frontiers, Minneapolis, Minn., USA) and 2-1 haptoglobin (Alpha Therapeutics Corporation, Chicago, Ill., USA) samples were prepared and run as standards on sodium dodecyl sulphate-polyacrylamide gels [15]. Both samples and molecular weight markers (BioRad Laboratories, Richmond, Calif., USA) were loaded and electrophoresed at a constant amperage until $5 \mathrm{~min}$ after the tracking dye exited the gels. Gels were processed by fixing in $50 \%$ methanol and $10 \%$ ace tic acid for $1 \mathrm{~h}$, staining in $0.05 \%$ Coomassic Brilliant Blue G-250 (Serva, Paramus, NJ, USA) in $5 \%$ perchloric acid for $20 \mathrm{~min}$, and destaining in two changes of $7 \%$ acetic acid and $5 \%$ methanol. All gels were dried under vacuum, photographed, and evaluated for haptoglobin Hp 1-1, Hp 2-1, and Hp 2-2 genotypes.

\section{Statistical analysis}

Statistical analyses were performed using chi-square tests of association. Since specific HLA antigens have been implicated in the pathogenesis of Type 1 diabetes, only the three HLA-B and two HLA-DR antigens were tested so that no correction for multiple antigen comparisons is required. Sib-pair analyses were used as a procedure to screen for linkage of HLA and haptoglobin with Type 1 diabetes in all families $[16,17]$. The sib-pair analysis reduces to a twosample $t$-test between trait-concordant and trait-discordant sibships, with respect to the mean proportion of genes identical by descent (IBD) at the marker [18]. The dependent variable is the probability that a sibling pair is discordant for diabetic susceptibility, which is a function of the age of each individual (sibling) and the mean and variance of the age of onset distribution. The regression coefficient for the probability that a sib-pair is discordant on the proportion of genes IBD at a marker is then estimated and tested for significance by a standard $t$-test. The average proportion of marker genes IBD for trait concordant sib-pairs (diabetic/diabetic and nondiabetic/non-diabetic) should be greater than half (the expected value under the assumption of no linkage). Similarly, the average proportion of marker genes IBD for trait discordant sib-pairs (diabetic/non-diabetic) should be less than half.

\section{Results}

The age at onset and sex distributions of Type 1 diabetes probands in Type 1 diabetes simplex and Type 1 diabetes multisib families are similar to that observed in other studies of Type 1 diabetes. The sex distribution of Type 1 diabetes probands in the Type 1 diabetes simplex (46 males:52 females) and Type 1 diabetes multisib (22 males:14 females) families does not differ greatly from the expected 1:1 ratio. However, the sex ratio of Type 1 diabetes probands in the Type 1/Type 2 families ( 9 males:19 females) differed significantly $(p<0.05)$ from that observed in most Type 1 diabetes proband studies. Further, the age at onset distribution of the Type 1 diabetes probands in the Type 1/Type 2 diabetes families $(18.3 \pm 2.0$ years $)$ is significantly $(p<0.05)$ older than in either the Type 1 diabetes simplex ( $11.5 \pm 0.8$ years) or the Type 1 diabetes multisib ( $11.6 \pm 1.1$ years) families. The difference in age at onset and sex distribution of the Type 1 diabetes probands in Type 1/Type 2 diabetes families may be due either to genetic heterogeneity or chance (small numbers).

\section{Association}

Distributions of tested HLA antigen frequencies in Type 1 probands and local control subjects and estimates of relative risks for Type 1 diabetes are given in Table 1. 
In the Type 1 simplex probands, there are statistically significant $(p<0.05)$ elevated relative risks $(\mathrm{RR})$ for HLA-B8 $(R R=2.31)$, HLA-B15 $(R R=2.41)$, HLA-B40 $(\mathrm{RR}=2.10)$, HLA-DR3 $(\mathrm{RR}=3.21)$ and HLA-DR4 $(\mathrm{RR}=5.09)$. These results are in agreement with previous studies and are partially the result of linkage disequilibrium which exists between HLA-B and HLA-DR alleles (B8-DR3, B15-DR4, and B40-DR4). In the Type 1 multisib probands, statistically significant elevated relative risks were also seen for HLA-B8 $(\mathrm{RR}=2.69)$, HLA-B15 $(\mathrm{RR}=3.65), \quad$ HLA-DR3 $\quad(\mathrm{RR}=2.74)$ and HLA-DR4 ( $R R=3.09)$. These HLA antigens, with the exception of HLA-B40, are the same and of the same magnitude of risk as those increased in the Type 1 simplex probands. The similarity in high risk antigens and magnitude of risk may indicate relative homogeneity in genetic susceptibility between Type 1 simplex and Type 1 multisib probands. In Type 1/Type 2 probands, significantly elevated relative risks are found for HLA-B15 $(\mathrm{RR}=2.37)$, HLA-B44 $(\mathrm{RR}=2.10)$, and HLA-DR4 $(\mathrm{RR}=4.32)$. In this subset of Type 1 diabetic patients those with at least one parent with Type 2 diabetes there is an absence of susceptibility due to the HLA B8DR3 haplotype. Only DR4-linked haplotypes, B15-DR4 and B44-DR4, appear to be associated with increased risk for Type 1 diabetes in families with at least one Type 2 diabetes parent.

Distributions of haptoglobin genotypes in Type 1 probands and local control subjects are given in Table 2 . In these patients, there is no significant association between a specific haptoglobin genotype and Type 1 diabetes. There is an excess of the haptoglobin $\mathrm{Hp}-1$ allele in the patients from the Type 1 simplex families, but the distribution of haptoglobin genotypes does not differ significantly from that of our local control subjects $\left(\chi_{2}^{2}=4.05, p=0.13\right)$. These data indicate that there is little evidence suggesting that a specific haptoglobin genotype or allele is associated with Type 1 diabetes.

Table 1. HLA allele frequencies and relative risk (RR) estimates in Type 1 (insulin-dependent) diabetic probands and control subjects partitioned by family type

\begin{tabular}{|c|c|c|c|c|c|c|c|}
\hline \multirow{2}{*}{$\begin{array}{l}\text { HLA } \\
\text { antigen }\end{array}$} & \multicolumn{2}{|c|}{ Type 1 simplex } & \multirow{2}{*}{\multicolumn{2}{|c|}{$\frac{\text { Type } 1 \text { multisib }}{\text { frequency RR }}$}} & \multirow{2}{*}{\multicolumn{2}{|c|}{$\frac{\text { Type 1/Type } 2}{\text { frequency RR }}$}} & \multirow{3}{*}{$\begin{array}{l}\text { Control } \\
\text { frequency }\end{array}$} \\
\hline & frequency & $\overline{\mathrm{RR}}$ & & & & & \\
\hline$\overline{\mathrm{B} 8}$ & 0.21 & $2.31^{\mathrm{b}}$ & 0.24 & $2.69^{\mathrm{b}}$ & 0.17 & 1.74 & \\
\hline B15 & 0.15 & $2.41^{b}$ & 0.21 & $3.65^{\mathrm{b}}$ & 0.15 & $2.37^{\mathrm{a}}$ & 0.07 \\
\hline B40 & 0.11 & $2.10^{\mathrm{b}}$ & 0.08 & 1.61 & 0.06 & 1.27 & 0.06 \\
\hline B44 & 0.09 & 0.72 & 0.07 & 0.58 & 0.23 & $2.10^{\mathrm{a}}$ & 0.12 \\
\hline DR3 & 0.31 & $3.21^{b}$ & 0.28 & $2.74^{\mathrm{b}}$ & 0.17 & 1.51 & 0.12 \\
\hline DR4 & 0.54 & $5.09^{b}$ & 0.42 & $3.09^{\mathrm{b}}$ & 0.58 & $4.32^{\mathrm{b}}$ & 0.19 \\
\hline
\end{tabular}

Table 2. Haptoglobin frequencies in Type 1 (insulin-dependent) diabetic probands and control subjects partitioned by family type

\begin{tabular}{lllll}
\hline $\begin{array}{l}\text { Haptoglobin } \\
\text { genotype }\end{array}$ & $\begin{array}{l}\text { Type 1 simplex } \\
\text { frequency }\end{array}$ & $\begin{array}{l}\text { Type 1 multisib } \\
\text { frequency }\end{array}$ & $\begin{array}{l}\text { Type 1/Type 2 Control } \\
\text { frequency }\end{array}$ & frequency \\
\hline $1-1$ & 0.20 & 0.19 & 0.18 & 0.18 \\
$2-1$ & 0.49 & 0.45 & 0.32 & 0.39 \\
$2-2$ & 0.31 & 0.36 & 0.50 & 0.43 \\
\hline
\end{tabular}

Table 3. Linkage between Type 1 (insulin-dependent) diabetes, HLA and haptoglobin

\begin{tabular}{llcrc}
\hline Family type & HLA & \multicolumn{3}{c}{ Haptoglobin } \\
\cline { 2 - 5 } & T-value & $p$-value & T-value & $p$-value \\
\hline Type 1/Type 2 & 2.761 & 0.0034 & 0.800 & 0.2128 \\
Type 1 simplex & 2.905 & 0.0019 & -1.628 & 0.9478 \\
Type 1 multisib & 3.539 & 0.0002 & -0.381 & 0.6484 \\
\hline
\end{tabular}

\section{Linkage}

A susceptibility locus for Type 1 diabetes may be linked to a genetic marker locus without demonstrating an association. In order to test whether a Type 1 diabetes susceptibility locus is linked to the haptoglobin locus, rather than associated with a haptoglobin genotype, a robust sib-pair linkage test was performed. Linkage with HLA was also tested in order to confirm previous results and to serve as an internal control for consistency. In the 27 families with a Type 1 diabetes proband and at least one Type 2 diabetes parent, there were a total of 184 sibling pairs available for analysis. Results of the sib-pair analysis (Table 3) indicate that there is significant evidence for linkage of Type 1 diabetes susceptibility with HLA in these families $(p=0.0034)$ but no evidence for linkage of Type 1 diabetes susceptibility with haptoglobin $(p=0.2128)$. In the 98 families with a single Type 1 diabetic child (proband) and non-diabetic parents, there were 568 sibling pairs available for analysis. There was significant evidence for linkage of Type 1 diabetes susceptibility with HLA $(p=0.0019)$ but no evidence for linkage of Type 1 diabetes susceptibility with haptoglobin $(p=0.9478)$. In the 36 families with at least two Type 1 diabetic children (one a proband) and non-diabetic parents, there were 447 sibling pairs available for analysis. There was significant evidence for linkage of Type 1 diabetes susceptibility with HLA $(p=0.0002)$ but no evidence for linkage of Type 1 diabetes susceptibility with haptoglobin $(p=0.6484)$. Thus, these results consistently demonstrate the presence of an HLA-linked Type 1 diabetes susceptibility factor and an absence of a haptoglobin-linked susceptibility factor.

\section{HLA-DR4 transmission}

One measure of the importance of HLA-DR4 in families with a Type 1 diabetes proband and at least one Type 2 diabetes parent is the frequency of transmission of HLADR4 from Type 2 parent to Type 1 child. If HLA-DR4 is contributing to Type 1 diabetes greater in degree than expected, then HLA-DR4-positive Type 1 diabetic patients would be expected to have received the HLA-DR4 from their Type 2 diabetic parent more often than expected based upon Mendelian segregation. In the families with a Type 1 diabetes proband and at least one Type 2 diabetes parent which were typed for HLA-DR, a total of 11 families had both parents HLA-typed and had a Type 1 diabetic subject who was HLA-DR4-positive. In these 11 Type 1 diabetic subjects, the HLA-DR4 allele was con- 
tributed by the Type 2 diabetic parent in 9 cases ( $82 \%$ ), significantly $(p<0.05)$ higher than expected under Mendelian segregation $(50 \%)$.

\section{Discussion}

A common theme in diabetes research has been that Type 1 diabetes is aetiologically distinct from Type 2 diabetes. Type 1 diabetes is considered to be autoimmune and is clearly associated with specific HLA antigens and demonstrates linkage of a major susceptibility locus with the HLA complex [19-22]. Type 2 diabetes is thought not to be autoimmune and has not shown a consistent association or linkage with genes in the HLA region. On the other hand, there is an increased risk for Type 1 diabetes in siblings when a Type 1 diabetic proband has a Type 2 diabetic parent $[4,5]$. We present evidence here that the genetic relationship between Type 1 and Type 2 diabetes may be accounted for, in part, through the HLA-DR4 antigen.

A popular hypothesis is that separate major genes are responsible for Type 1 and Type 2 susceptibility. These major locus models are attractive for several reasons, the primary one being that a major locus has a high probability of being mapped to a chromosomal location. Although genetic susceptibility to Type 1 diabetes is strongly influenced by genes in the HLA region of human chromosome 6 , not all of the susceptibility is accounted by this one region. This can be seen by the observation that $55 \%$ of trait concordant sibling pairs (both siblings have Type 1 diabetes), will have the same HLA region genes (haplotypes), $40 \%$ of the sibling pairs will have only one haplotype in common, while $5 \%$ of the sibling pairs will have no HLA haplotypes in common [23]. Although a multifactorial source of genetic liability to Type 1 diabetes and/or Type 2 diabetes is a concern for gene mapping, there is no need to imply that multifactorial inheritance implies hundreds of loci. Rather, there remains the more likely outcome that Type 1 and Type 2 diabetes are each the result of the actions of a limited number of genes (a major gene and some genes with small effect). Thus, of the additional loci required for at least some forms of Type 1 diabetes and Type 2 diabetes, some overlap may occur.

Several studies have documented the increased Type 1 diabetes risk to siblings of a Type 1 diabetic proband when a parent has Type 2 diabetes $[4,5]$. This increase in risk is similar in magnitude to that seen for siblings of a Type 1 diabetes proband when a parent has Type 1 diabetes. With multiple loci required for both Type 1 diabetes and Type 2 diabetes, the possibility exists that a single locus may contribute to the susceptibility to both diseases. A genetic relationship between susceptibility for Type 1 diabetes and Type 2 diabetes would allow for a resolution between the models which have been proposed to explain the observed clinical heterogeneity and expression of both Type 1 diabetes and Type 2 diabetes. Recent studies [24] have demonstrated an increased serum viscosity in Type 2 diabetes patients with microvascular disease which has been linked to changes in serum protein composition. The major change observed is a decline in albumin and an in- crease in the levels of acute-phase proteins (including haptoglobin). Acute-phase proteins also were increased in advanced glucose intolerance as well as in established Type 2 diabetes, although there was no relationship with degree of control or duration of diabetes. Although this effect may be due to metabolic changes (increased blood glucose), the examination of families with both Type 2 and Type 1 diabetes with respect to genetic markers could be highly informative in defining mutual risk factors.

HLA antigen distributions in the Micronesian population of Nauru [25] showed a small increased risk for early onset of diabetes (less than 46 years) associated with HLA-Bw22 (Bw56). In a series of studies [26,27], islet cell antibodies (ICAs), thyrogastric antibodies, and HLA-DR antigens were determined in 204 patients with Type 2 diabetes controlled with diet and/or oral hypoglycaemic agents and in 108 age-matched patients who required insulin to control their hyperglycaemia. The insulin-requiring patients differed from the non-insulin-requiring patients with respect to higher frequency of ICAs, thyroid antibodies, and HLA-DR4 frequency. Patients who were heterozygous for HLA-DR3/DR4 showed significantly higher frequency of ICAs and complement-fixing ICAs than patients without the heterozygous form DR3/DR4. These data suggested that Type 2 diabetes is a heterogeneous disorder including at least two major subgroups, characterized by HLA-DR antigens and organ-specific antibodies.

The role of genetic markers other than HLA in contributing to susceptibility to both Type 1 diabetes and Type 2 diabetes has been equivocal. In a Mexican American population randomly selected from three San Antonio neighbourhoods, statistically significant associations were found between Type 2 diabetes and the Rhesus blood type and haptoglobin phenotype [28, 29]. The haptoglobin association showed a dose effect with a single dose of the Hp-1 allele associated with a $50 \%$ increased Type 2 diabetes prevalence and a HP 1-1 homozygote was associated with a $100 \%$ increase in Type 2 diabetes prevalence. In a separate study of association between Type 2 diabetes and seven polymorphic markers using 398 Caucasian subjects (97 Type 2 diabetes and 301 normal individuals) and 390 Hispanic subjects (191 Type 2 diabetes and 199 normal individuals), there was no association with either haptoglobin or $\mathrm{Rh}$ [30]. In our families with at least one Type 2 diabetic parent and a Type 1 diabetic child, the haptoglobin genotype does not appear to be a significant contributor to diabetes susceptibility, either with respect to association with diabetes or with respect to linkage with a secondary susceptibility locus.

Haptoglobin phenotyping in patients at risk for myocardial infarction and essential hypertension have previously demonstrated a significant increase in the incidence of the $\mathrm{Hp} 2-2$ phenotype in the high-risk group, as compared to a group of normal subjects $[31,32]$. Since there is a significant risk of cardiovascular events in patients with Type 2 diabetes [33], existence of a mechanism involving haptoglobin (either plasma levels or genotypes) in the pathogenesis of cardiovascular disease may be sufficient to appear important in subsets of Type 2 diabetes patients. 
The possible relationship between haptoglobin phenotype and Type 1 diabetes, insulin antibodies and microangiopathy has been previously studied [34]. There were no differences regarding the distribution of haptoglobin phenotypes in patients in comparison with control subjects. There also were no relationships between haptoglobin genotype and diabetic microangiopathy (retinopathy, nephropathy) or insulin antibody binding. However, both the maximum insulin binding capacity and the equilibrium dissociation constant were found to vary considerably with the haptoglobin genotype among Type 1 diabetic patients. Differences between insulin binding parameters, reflecting differences in haptoglobin genotype, may be of clinical importance.

In summary, we present data which is consistent with Type 1 and Type 2 diabetes sharing factor(s) responsible for genetic susceptibility in at least some families. The data supportive of this contention include the previously reported significantly increased cumulative risk for Type 1 diabetes in siblings of Type 1 probands with a Type 2 diabetic parent and the increased relative risk for Type 1 diabetes associated with HLA-DR4 but not HLADR3 in these families. These results provide evidence that families with a Type 2 parent and Type 1 child, heavily determined by HLA-DR4 linked factors, may represent a homogeneous subset of diabetes susceptibility.

Acknowledgements. We thank Dr. L. R. Weitkamp for early support in this study and for providing early haptoglobin typing data, Dr. J.Reusch for contacting the patients and evaluating family members, Ms. A. Martinez for expert database support, and Dr. M. Segall for evaluation of the manuscript. This study was supported in part by grants from the Eli Lilly and Company, Indianapolis, Ind., the Graduate School of the University of Minnesota, the American Diabetes Association, Minnesota Affiliate, and by NIH grants AM20729, DK36828, DK33225, NS16308, RR03655, and RR00400 (General Clinical Research Center).

\section{References}

1. Barnett AH, Eff C, Leslie RD, Pyke DA (1981) Diabetes in identical twins. A study of 200 pairs. Diabetologia 20: 87-93

2. Olmos P, A'Hern R, Heaton DA, Millward BA, Risley D, Pyke DA, Leslie RD (1988) The significance of the concordance rate for Type 1 (insulin-dependent) diabetes in identical twins. Diabetologia 31: 747-750

3. Newman B, Selby JV, King MC, Slemenda C, Fabsitz R, Friedman GD (1987) Concordance for Type 2 (non-insulin-dependent) diabetes mellitus in male twins. Diabetologia 30: 763-768

4. Chern MM, Anderson VE, Barbosa J (1982) Empirical risk for insulin-dependent diabetes (IDD) in sibs. Further definition of genetic heterogeneity. Diabetes 31:1115-1118

5. Wagener DK, Sacks JM, LaPorte RE, Macgregor JM (1982) The Pittsburgh study of insulin-dependent diabetes mellitus. Risk for diabetes among relatives of IDDM. Diabetes 31: 136 144

6. Thomson G (1980) A two locus model for juvenile diabetes. Ann Hum Genet 43: 383-398

7. Hodge SE, Rotter JI, Lange KL (1980) A three-allele model for heterogeneity of juvenile onset insulin-dependent diabetes. Ann Hum Genet 43: 399-409

8. Clerget DF, Bonaiti PC, Deschamps I, Hors J, Feingold N (1981) Juvenile insulin-dependent diabetes: a possible susceptibility gene in interaction with HLA. Ann Hum Genet 44: 195204
9. Field LL, Anderson CE, Neiswanger K, Hodge SE, Spence MA, Rotter JI (1984) Interaction of HLA and immunoglobulin antigens in Type 1 (insulin-dependent) diabetes. Diabetologia 27: $504-508$

10. National Diabetes Data Group (1979) Classification and diagnosis of diabetes mellitus and other categories of glucose intolerance. Diabetes 28: 1039-1054

11. Rich SS, Weitkamp LR, Barbosa J (1984) Genetic heterogeneity of insulin-dependent (Type I) diabetes mellitus: evidence from a study of extended haplotypes. Am J Hum Genet 36: 10151023

12. French LR, Boen JR, Martinez AM, Bushhouse SA, Sprafka JM, Goetz FC (1990) A population-based study of impaired glucose tolerance and Type II diabetes: The Wadena City Health Study. Diabetes 39:1131-1137

13. Barbosa J, Bach FH, Rich SS (1982) Genetic heterogeneity of diabetes and HLA. Clin Genet 21:25-32

14. Roy RB, Shaw RW, Connell GE (1969) A simple method for the quantitative determination of serum haptoglobin. J Lab Clin Med 74: 698-704

15. O'Farrell PH (1975) High resolution two-dimensional electrophoresis of proteins. J Biol Chem 250: 4007-4021

16. Blackwelder WC, Elston RC (1985) A comparison of sib-pair linkage tests for disease susceptibility loci. Genet Epidemiol 2: $85-97$

17. Amos CI, Elston RC, Wilson AF, Bailey-Wilson JE (1989) A more powerful robust sib-pair test of linkage for quantitative traits. Genet Epidemiol 6: 435-449

18. Elston RC, Stewart J (1971) A general model for the genetic analysis of pedigree data. Hum Hered 21: 523-542

19. Nerup J, Platz P, Andersen OO, Christy MM, Lyngsoe J, Poulsen JE, Ryder LP, Nielsen LS, Thomsen M, Svejgaard A (1974) HL-A antigens and diabetes mellitus. Lancet II: 864-866

20. Thomsen M, Platz P, Andersen OO, Christy M, Lyngsoe, Nerup J, Rasmussen K, Ryder LP, Nielsen LS, Svejgaard A (1975) MLC typing in juvenile diabetes mellitus and idiopathic Addison's disease. Transplant Rev 22: 125-147

21. Barbosa J, King R, Noreen H, Yunis EJ (1977) The histocompatibility system in juvenile, insulin-dependent diabetic multiplex kindreds. J Clin Invest 60: 989-998

22. Svejgaard A, Jakobsen BK, Platz P, Ryder LP, Nerup J, Christy M, Borch JK, Parving HH, Deckert T, Molsted PL, Kuhl C, Buschard K, Green A (1986) HLA associations in insulin-dependent diabetes: search for heterogeneity in different groups of patients from a homogeneous population. Tissue Antigens 28: 237-244

23. Spielman RS, Baker L, Zmijewski CM (1980) Gene dosage and susceptibility to insulin-dependent diabetes. Ann Hum Genet 44: $135-150$

24. McMillan DE (1989) Increased levels of acute-phase serum proteins in diabetes. Metabolism 38: 1042-1046

25. Serjeantson SW, Owerbach D, Zimmet P, Nerup J, Thoma K (1983) Genetics of diabetes in Nauru: effects of foreign admixture, HLA antigens and the insulin-gene-linked polymorphism. Diabetologia 25: 13-17

26. Groop L, Koskimies S, Pelkonen R, Tolppanen EM (1983) Increased frequency of HLA-Cw4 in type 2 diabetes. Acta Endocrinol (Copenh) 104: 475-478

27. Groop L, Miettinen A, Groop PH, Meri S, Koskimies S, Bottazzo GF (1988) Organ-specific autoimmunity and HLA-DR antigens as markers for beta-cell destruction in patients with type II diabetes. Diabetes 37: 99-103

28. Stern MP, Ferrell RE, Rosenthal M, Haffner SM, Hazuda HP (1986) Association between NIDDM, RH blood group, and haptoglobin phenotype. Results from the San Antonio Heart Study. Diabetes 35: 387-391

29. Chakraborty R, Ferrell RE, Stern MP, Haffner SM, Hazuda HP, Rosenthal M (1986) Relationship of prevalence of non-insulindependent diabetes mellitus to Amerindian admixture in the Mexican Americans of San Antonio, Texas. Genet Epidemiol 3: $435-454$ 
30. Iyengar S, Hamman RF, Marshall JA, Baxter J, Majumder PP, Ferrell RE (1989) Genetic studies of Type 2 (non-insulin-dependent) diabetes mellitus: lack of association with seven genetic markers. Diabetologia 32: 690-693

31. Gogishvili AV, Kavtaradze VG, Mamaladze GT, Arutiunova MS, Takadze G (1985) Haptoglobin phenotype distribution in patients at high risk of developing myocardial infarct. Kardiologiia 25: $55-58$

32. Surya PP, Padma T, Ramaswamy M (1987) Haptoglobin patterns in essential hypertension and associated conditions-increased risk for $\mathrm{Hp} 2-2$. Hum Hered 37: 345-348

33. Mandrup PT, Owerbach D, Nerup J, Johansen K, Tybjaerg HA (1986) Diabetes mellitus, atherosclerosis, and the 5' flanking polymorphism of the human insulin gene. J Inherited Metab Dis 1: $98-110$
34. Ratzmann KP, Strese J, Keilacker H, Giebelmann R, Scheibe F, Witt $S$ (1984) Is there a relationship between genetically determined haptoglobin phenotype and insulin-dependent diabetes mellitus (IDDM)? Exp Clin Endocrinol 83: 207-215

Received: 17 September 1990

and in revised form: 2 January 1991

Dr. S.S.Rich

Box 511 U. M.H.C.

Department of Laboratory Medicine and Pathology

University of Minnesota

Minneapolis, MN 55455

USA 